\title{
The triple system HIP 96515: a low-mass eclipsing binary with a DB white dwarf companion ${ }^{\star}$
}

\author{
N. Huélamo ${ }^{1}$, L. P. R. Vaz ${ }^{2}$, C. A. O. Torres ${ }^{3}$, P. Bergeron ${ }^{4}$, C. H. F. Melo ${ }^{5}$, G. R. Quast ${ }^{3}$, D. Barrado y Navascués ${ }^{1}$, \\ M. F. Sterzik ${ }^{6}$, G. Chauvin ${ }^{7}$, H. Bouy ${ }^{8, \star \star}$, and N. R. Landin ${ }^{2}$ \\ 1 LAEX-CAB (INTA-CSIC); Postal address: LAEFF, PO Box 78, 28691 Villanueva de la Cañada, Madrid, Spain \\ e-mail: nhuelamo@laeff.inta.es \\ 2 Depto. de Física, Universidade Federal de Minas Gerais, C.P.702, 30161-970 - Belo Horizonte, MG, Brazil \\ 3 Laboratório Nacional de Astrofísica/MCT, Rua Estados Unidos 154, 37504-364 Itajubá, Brazil \\ 4 Département de Physique, Université de Montréal, C.P. 6128, Succ. Centre-Ville, Montréal, Québec H3C 3J7, Canada \\ 5 European Southern Observatory, Karl-Schwarzschild-Strasse 2, 85748 Garching bei Muenchen, Germany \\ ${ }^{6}$ European Southern Observatory, Alonso de Cordova 3107, Casilla 19, Santiago, Chile \\ 7 Laboratoire d'Astrophysique, Observatoire de Grenoble, BP 53, 38041 Grenoble, Cedex 9, France \\ 8 Instituto de Astrofísica de Canarias, C Vía Láctea, s/n, E38205 - La Laguna (Tenerife), Spain
}

Received 11 December 2008 / Accepted 27 January 2009

\section{ABSTRACT}

\begin{abstract}
Context. HIP $96515 \mathrm{~A}$ is a double-lined spectroscopic binary included in the SACY catalog as a potential young star. It has a visual companion (CCDM 19371-5134 B, HIP 96515 B) at 8".6. If bound to the primary, the optical and infrared colors of this wide companion are consistent with those of a white dwarf.

Aims. We attempt to characterize the system HIP 96515 A\&B by studying each of its components.

Methods. We analyzed spectroscopic and photometric observations of HIP 96515 A and its visual companion, HIP 96515 B. To confirm the system as a common proper-motion pair, we analyzed the astrometry of the components using high-angular resolution infrared observations obtained within a time span of two years, and archival astrometry.

Results. The high-resolution optical spectrum of HIP 96515 A was used to derive a mass ratio, $M_{2} / M_{1}$, close to 0.9. The optical lightcurve of HIP 96515 A shows periodic variations with $P_{\text {orbital }}=2.3456$ days, revealing that HIP 96515 A is an eclipsing binary with preliminary orbital parameters of $i=89.0 \pm 0.2$, and $M_{1}=0.59 \pm 0.03 M_{\odot}$ and $M_{2}=0.54 \pm 0.03 M_{\odot}$, for the primary and secondary, respectively, at an estimated distance of $42 \pm 3 \mathrm{pc}$. This is a new eclipsing binary with component masses below $0.6 M_{\odot}$. Multi-epoch observations of HIP 96515 A\&B show that the system is a common proper-motion pair. The optical spectrum of HIP 96515 B is consistent with a pure helium atmosphere (DB) white dwarf. The comparison with evolutionary cooling sequence models provides $T_{\text {eff,WD }}=19126 \pm 195 \mathrm{~K}, \log g_{\mathrm{WD}}=8.08, M_{\mathrm{WD}} / M_{\odot}=0.6$, and a distance of $\sim 46 \mathrm{pc}$. The estimated WD cooling age is $\sim 100 \mathrm{Myr}$ and the total age of the object (including the main-sequence phase) is $\sim 400 \mathrm{Myr}$. Finally, if HIP 96515 A\&B are coeval, and assuming a common age of $\sim 400 \mathrm{Myr}$, the comparison of the masses of the eclipsing binary members with evolutionary tracks shows that they are underestimated by $\sim 15 \%$ and $10 \%$, for the primary and secondary, respectively.
\end{abstract}

Key words. stars: white dwarfs - stars: binaries: eclipsing - stars: individual: HIP 96515

\section{Introduction}

In recent years, several infrared surveys have focused on the detection of substellar companions around young and nearby stars using high-contrast imaging techniques, (e.g., Chauvin et al. 2003; Lowrance et al. 2005; Masciadri et al. 2005; Lafreniere et al. 2007). Young nearby stars are privileged targets for these kind of studies due to their proximity (below $100 \mathrm{pc}$ ), which allows to study very small separations, and their youth, which enhances the contrast between the central star and the potential companion.

In 2006, we started an infrared survey to detect substellar objects around young stars from the SACY catalog (Torres et al. 2003, 2006). Our sample included HIP 96515, a nearby $(d=$ $43.9 \pm 9$ pc, van Leeuwen 2007) M1-type star classified as a double-lined spectroscopic binary (SB2) by Torres et al. (2006).

\footnotetext{
* Based on observations collected at the Paranal Observatory under programs 77.C-0483(A) and 81.C-0826(A).

$\star \star$ Marie Curie Outgoing International Fellow.
}

The object is catalogued as a visual binary (CR23, Worley \& Douglass 1997), with a secondary companion at an angular separation of $\rho \sim$ 8.'6 (CCDM 19371-5134B or HIP 96515 B Dommanget \& Nys 2000). This visual binary has been detected in different infrared and optical surveys, although its properties have never been studied in detail.

As a first step in unveiling the nature of HIP $96515 \mathrm{~B}$, we compared our $K_{\mathrm{s}}$-band photometry with evolutionary tracks (Baraffe et al. 2003; Chabrier et al. 2000). The comparison suggested that HIP 96515 B may be of substellar nature if the age of the primary is in the range 0.07-1 Gyr and the two objects are comoving. However, the optical and infrared colors retrieved from archival observations are inconsistent with being a substellar object, but consistent with those of a white dwarf.

Motivated by this preliminary result, we decided to perform a detailed study of HIP 96515. We analyzed new and archival observations of the triple system and derived the main properties of each source. Section 2 describes the main observational properties of HIP 96515 A\&B. In Sect. 3, we analyze spectroscopic 
Table 1. Main properties of HIP 96515 A.

\begin{tabular}{|c|c|c|c|c|c|}
\hline \multirow{2}{*}{$\alpha(\mathrm{J} 2000)$} & \multirow{2}{*}{$\delta(\mathrm{J} 2000)$} & Spectral & Parallax $^{1}$ & $\mu_{\alpha}^{1}$ & $\mu_{\delta}^{1}$ \\
\hline & & type & [mas] & {$\left[\right.$ mas $\left.\mathrm{yr}^{-1}\right]$} & {$\left[\mathrm{mas} \mathrm{yr}^{-1}\right]$} \\
\hline $19^{\mathrm{h}} 37^{\mathrm{m}} 08^{\mathrm{s}} .7$ & $-51^{\circ} 34^{\prime} 00^{\prime} 9$ & $\mathrm{M} 1^{2}$ & $\begin{array}{l}22.79 \\
\pm 4.87\end{array}$ & $\begin{array}{r}91.07 \\
\pm 4.93\end{array}$ & $\begin{array}{r}-21.94 \\
\pm 4.62\end{array}$ \\
\hline
\end{tabular}

Notes: ${ }^{1}$ van Leeuwen (2007); ${ }^{2}$ Torres et al. (2006).

Table 2. Photometric data of the visual binary HIP 96515 A\&B.

\begin{tabular}{llcccl}
\hline \hline Target & $V$ & $R$ & $J$ & $H$ & $K_{\mathrm{s}}$ \\
& {$[\mathrm{mag}]$} & {$[\mathrm{mag}]$} & {$[\mathrm{mag}]$} & {$[\mathrm{mag}]$} & {$[\mathrm{mag}]$} \\
\hline HIP 96515 A & $11.78^{1}$ & $10.90^{1}$ & $8.82^{2}$ & $8.15^{2}$ & $7.96^{2}$ \\
& \pm 0.04 & \pm 0.03 & \pm 0.03 & \pm 0.04 & \pm 0.03 \\
HIP 96515 B & $13.0^{3}$ & $14.3^{4}$ & $14.54^{2}$ & $14.44^{2}$ & $14.52^{2}, 14.94^{5}$ \\
& & \pm 0.3 & \pm 0.18 & \pm 0.51 & $\pm 0.31 \pm 0.08$ \\
\hline
\end{tabular}

Notes: ${ }^{1}$ Torres et al. (2006); ${ }^{2}$ 2MASS catalog (Cutri et al. 2003); ${ }^{3}$ Visual Double stars in Hipparcos catalog (Dommanget \& Nys 2000);

${ }^{4}$ UCAC2 catalog; ${ }^{5}$ NACO/VLT data (this work).

and photometric data of the primary star HIP $96515 \mathrm{~A}$, while the true nature of the visual component, HIP 96515 B is unveiled in Sect. 4. A discussion of the system is provided in Sect. 5, and the main results are summarized in Sect. 6.

\section{Observational properties of HIP 96515 A\&B}

The main stellar properties of HIP $96515 \mathrm{~A}$ are displayed in Table 1, while its optical and infrared photometry are shown in Table 2.

The analysis of the optical spectrum of HIP96515 A obtained during the SACY survey, allowed Torres et al. (2006) to classify the object as an SB2 with almost equal-mass components (see Table 3 ). From the cross-correlation function (CFF), we estimated a magnitude difference of $\Delta V=0.55$ mag between the binary components.

HIP 96515 was included in the ROS AT All-Sky Bright Star catalog (Voges et al. 1999). The source was detected by ROS AT with a count rate of $0.172 \pm 0.036$ counts $s^{-1}$. We derived the $\mathrm{X}$-ray flux using the conversion factor definition by Schmitt et al. (1995). The X-ray luminosity, assuming a distance of $44 \mathrm{pc}$ (see Table 1), is $L_{\mathrm{x}}=(3.61 \pm 0.75) \times 10^{29} \mathrm{erg} \mathrm{s}^{-1}$. Using the bolometric correction $(B C)$ by Kenyon \& Hartmann (1995) for an M1-type star, and the $V$-mag listed in Table 2, we derived a $\log \left(L_{\mathrm{X}} / L_{\mathrm{bol}}\right)$ ratio of -3.07 . If we assume that the two components of the spectroscopic binary (HIP $96515 \mathrm{Aa} \& \mathrm{Ab}$ ) emit in X-rays, such that $L_{x, A a}=L_{x, A b}=L_{x} / 2$, and using the $V$-mag of each of the binary members (see Table 3 ) and the $B C$ for a M1 and M2 star, we derive $\log \left(L_{\mathrm{x}} / L_{\mathrm{bol}}\right)$ ratios of -3.17 and -3.04 for $\mathrm{Aa}$ and $\mathrm{Ab}$, respectively. These values are comparable to those found in very active pre-main sequence (PMS) late-type stars (e.g., Barrado y Navascués et al. 1999; Stelzer \& Neuhäuser 2001) and in main-sequence (MS) late-type stars in very close binaries (see e.g. López-Morales 2007).

The age of HIP96515 A is unknown. The lithium content is normally used as a youth indicator in the case of late-type stars. However, it is not always present in young M-type stars since it is depleted too fast (e.g., Basri et al. 1996; Neuhäuser 1997; Torres et al. 2008a). In fact, none of the binary members of HIP 96515 A show the lithium absorption doublet $(\lambda=$ $6707.8 \AA$ ) in the optical spectrum. Torres et al. (2008a) studied
Table 3. Spectroscopic data of the eclipsing binary HIP 96515 A.

\begin{tabular}{llllll}
\hline \hline Star & $\begin{array}{l}V \\
{[\mathrm{mag}]}\end{array}$ & Spt. type & $\begin{array}{l}v_{\mathrm{rad}} \\
{\left[\mathrm{km} \mathrm{s}^{-1}\right]}\end{array}$ & $\begin{array}{l}\mathrm{H} \alpha E W \\
{[\AA]}\end{array}$ & $\begin{array}{l}v \sin i \\
{\left[\mathrm{~km} \mathrm{~s}^{-1}\right]}\end{array}$ \\
\hline $96515 \mathrm{Aa}$ & 12.29 & M1Ve & 84.1 & 0.9 & $14.8 \pm 1.5$ \\
$96515 \mathrm{Ab}$ & 12.84 & M2:Ve & -81.3 & 2.0 & $15.8 \pm 1.6$ \\
\hline
\end{tabular}

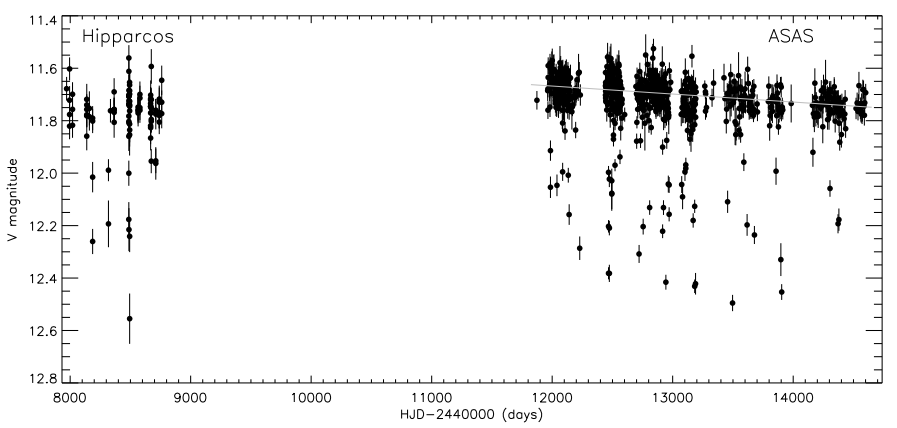

Fig. 1. Hipparcos and ASAS V-band light-curves of HIP 96515 A.

the lithium content as a function of the $V-I$ color in different young associations with ages in the range 5-70 Myr. By comparing HIP 96515 A with similar M-type stars (with $V-I \sim 1.8$, Torres et al. 2006) in those associations, we can compute a lower limit to the age of $\geq 70 \mathrm{Myr}$. By following the temporal evolution of lithium in stellar models (e.g., those by Landin et al. 2006) for stars with the masses of HIP 96515 A (Sect. 3), Li should be exhausted by an age between $30 \mathrm{Myr}$ (for a mixing length parameter of $\alpha=2$ ) and $50 \operatorname{Myr}($ for $\alpha=1$ ).

Finally, HIP 96515 B is included in the AC catalog (Urban et al. 1998) as a visual companion to HIP 96515 A. The object was detected in different infrared and optical surveys and multi-wavelength photometry is available in different public catalogs. We included all the available data in Table 2. We note that, in most cases, the photometric measurements show large uncertainties.

\section{Characterization of HIP 96515 A: a M1+M2 eclipsing binary}

To characterize the SB2 in more detail, we retrieved photometric data of HIP 96515 A from both the Hipparcos archive (Perryman et al. 1997, 96 data points) and the All Sky Automated Survey, $\mathrm{ASAS}^{1}$, (Pojmanski \& Maciejewski 2005, 818 data points). The Hipparcos observations, in its own photometric system (Hip), were corrected with the relation $V_{\mathrm{J}}=$ Hip -0.08 , suggested for the $V-I$ of this object ( $V-I=1.85$; Torres et al. 2006).

The observations of the source are displayed in Fig. 1, showing significant variability. The object was classified as an eclipsing binary by Paczyński et al. (2006), using ASAS data alone. We applied the method by Lafler \& Kinman (1965) to search for any periodicity in the data. The most likely period, 2.345 days, was used to phase-fold both the ASAS and Hipparcos data, confirming the SB2 as an eclipsing system. We now describe our full analysis of the photometric data and derive the main properties of the eclipsing components, Aa and Ab.

1 http://www.astrouw.edu.pl/ gp/asas/asas.html 


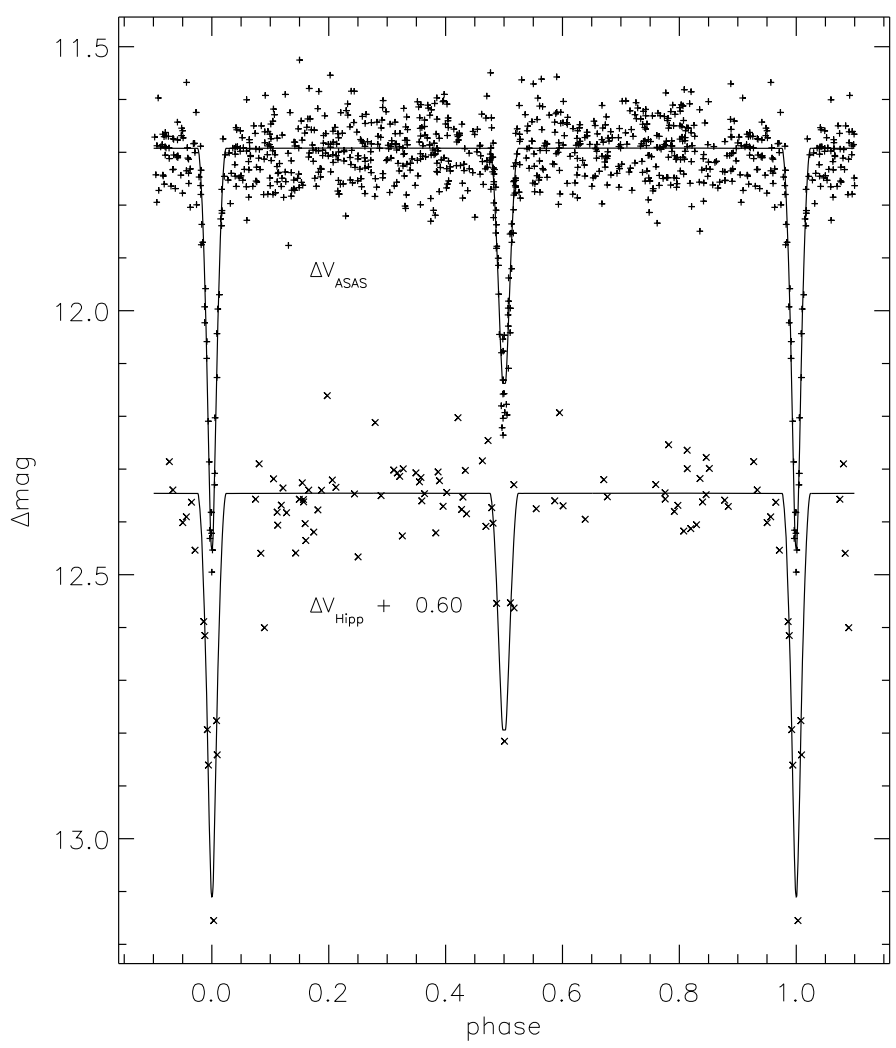

Fig. 2. The phase-folded lightcurves of HIP 96515 A from ASAS (+ symbols) and Hipparcos ( $\times$ symbols), consisting of 818 and 96 observations, respectively, with our theoretical solution for $q=0.9 \mathrm{ob}-$ tained in Sect. 3.2. The Hipparcos observations were shifted by $0.6 \mathrm{mag}$, in order to separate the light curves. Note that ASAS observations were not corrected from the long term variation of the maxima, shown in Fig. 1.

\subsection{Photometric data: period search}

The method by Lafler \& Kinman (1965) was used to search for periods in the Hipparcos $V$-band observations of HIP $96515 \mathrm{~A}$. We searched for periods between 0.5 day and 63 days, by using a relative period step of $\delta P / P=4 \times 10^{-7}$. Since $M$ stars may flare, and HIP 96515 is a strong X-ray emitter, we excluded the 4 brightest measurements (from Hipparcos measurements) in this period search, to avoid clearly non-periodic events, if present. Approximately 60 significant periods were found, but the most significant was $P_{\text {orb }}=(2.344451 \pm 0.000002) \mathrm{d}$. In the analysis of the photometry with a version of the WD model (see Sect. 3.2), we adjusted a phase shift to the folded observations, and obtained the ephemeris (HJD; the digits in parentheses affect the last digits)

Min I $=2448493.80515(10)+2.3444507(15) E$,

which we use in our analysis. According to Eq. (1), the secondary minimum occurs close to phase 0.5 , indicating an orbit with a small (if any) eccentricity, and wihout further evidence, we assume in the preliminary analysis a circular orbit. We note that circular orbits are consistent with short orbital (and rotational) periods, as found for HIP 96515 A (e.g., Zahn 1989). The $V$-band lightcurves (Hipparcos and ASAS), folded with these ephemeris, are shown in Fig. 2. The stellar eclipses are clearly seen in both datasets.

\subsection{Preliminary analysis of the HIP 96515 A light-curve}

We analyzed the Hipparcos optical lightcurve with a version of the WD model (Wilson \& Devinney 1971; Wilson 1979, 1993) extensively improved as described in Vaz et al. (2007) and references therein. Our version uses stellar atmospheres and models, the radiated flux of both components using the PHOENIX atmosphere models (Allard \& Hauschildt 1995; Allard et al. 1997; Hauschildt et al. 1997b,a). The (linear-law) limb-darkening coefficients for both components were taken from Claret (2000), and interpolated using a bilinear scheme for the current values of $\log g$ and $T_{\text {eff }}$ at each iteration. The reflection albedos for both components were fixed to a value of 0.5 , which is appropriate for stars with convective envelopes. The gravity-brightening exponent, $\beta$, was computed using the local value of $T_{\text {eff }}$ for each point on the stellar surface and taking into account mutual illumination following Alencar \& Vaz (1997) and Alencar et al. (1999). Our procedure combines the WD modified code with several UNIX scripts and auxiliary programs to guarantee the consistency of the solutions in all steps.

We have a single measurement of the radial velocity for each component at the phase 0.7715 , according to Eq. (1), by chance, close to the quadrature $(0.75$ in a circular orbit), when the line separation is maximum. Since we do not have any estimation of the center of mass velocity of the system, the mass ratio is undefined. Therefore, to investigate the solution space, we decided to compile a grid of solutions for different values of the mass ratio, $q\left(=M_{2} / M_{1}\right)$, varying from 1.000 to 0.850 in steps of 0.025 . In compiling this grid, we can determine the velocity of the center of mass of the system for each $q$, and adjust the remaining parameters to reproduce the individual heliocentric velocities measured at phase 0.7715 .

We assigned an arbitrary initial value of $89^{\circ}$ to the system inclination and used the WD model to find initial values of the gravitational pseudo-potentials (which define the size and form of the components) to reproduce the optical lightcurve. Finally, the initial values of the effective temperatures were adopted from the tabulated values listed in Popper (1980), and $T_{\text {eff,pri }}$ was kept fixed at the adopted value of $3714 \pm 150 \mathrm{~K}$. In the lack of further information, the eclipsing components were considered to be synchronized with the orbital motion, an assumption that may not be true (see below).

Despite the presence of a visual companion to HIP $96515 \mathrm{~A}$ at 8.6 , we do not expect any contamination in the Hipparcos lightcurve of the primary, since HIP $96515 \mathrm{~B}$ is outside the Hipparcos field of view. On the other hand, the resolution of the ASAS setup is 14".2/pixel and the light from HD $96515 \mathrm{~B}$ is definitely included in their measurements. Therefore, we assume a small amount of third light contribution in the analysis of the ASAS data lightcurve. From the appearance of the folded light curve (using our ephemeris) and in the absence of a well determined radial velocity curve, we assumed a circular orbit in our analysis.

Taking into account the afore mentioned initial values and assumptions, we modeled the orbital inclination, the gravitational pseudo-potentials, the secondary effective temperature, a phase shift in the primary minimum, and the primary internal luminosity of each point in the grid. The most interesting results from our modeling are displayed in Table 4.

After convergence of the solutions in Table 4, we adjusted the amount of third light in the $q=0.900$ solution, because of evidence that the visual companion, HIP $96515 \mathrm{~B}$, contaminates the ASAS measurements. In units of the eclipsing system light at quadrature, the third light parameter was determined 
Table 4. Grid of solutions for 7 values of the mass ratio, $q\left(=M_{2} / M_{1}\right)$, for HIP $96515 \mathrm{~A}$.

\begin{tabular}{lrrrrrrr}
\hline \hline$q\left(=M_{2} / M_{1}\right)$ & 1.000 & 0.975 & 0.950 & 0.925 & 0.900 & 0.875 & 0.850 \\
\hline$V_{1}\left(\mathrm{~km} \mathrm{~s}^{-1}\right)$ & 82.70 & 81.65 & 80.60 & 79.48 & 78.35 & 77.19 & 75.99 \\
$V_{2}\left(\mathrm{~km} \mathrm{~s}^{-1}\right)$ & -82.70 & -83.75 & -84.82 & -85.92 & -87.05 & -88.21 & -89.40 \\
$V_{\gamma}\left(\mathrm{km} \mathrm{s}^{-1}\right)$ & 1.40 & 2.45 & 3.52 & 4.62 & 5.75 & 6.91 & 8.10 \\
$K_{1}\left(\mathrm{~km} \mathrm{~s}^{-1}\right)$ & 83.46 & 82.41 & 81.32 & 80.21 & 79.07 & 77.90 & 76.69 \\
$K_{2}\left(\mathrm{~km} \mathrm{~s}^{-1}\right)$ & 83.46 & 84.51 & 85.60 & 86.71 & 87.85 & 89.02 & 90.23 \\
$M_{1}\left(M_{\odot}\right)$ & 0.56 & 0.57 & 0.58 & 0.59 & 0.59 & 0.60 & 0.61 \\
$R_{1}\left(R_{\odot}\right)$ & 0.64 & 0.65 & 0.65 & 0.64 & 0.64 & 0.64 & 0.64 \\
$\log g_{1}(\mathrm{cgs})$ & 4.57 & 4.57 & 4.58 & 4.59 & 4.60 & 4.60 & 4.61 \\
$M_{2}\left(M_{\odot}\right)$ & 0.56 & 0.56 & 0.55 & 0.54 & 0.53 & 0.53 & 0.52 \\
$R_{2}\left(R_{\odot}\right)$ & 0.53 & 0.53 & 0.53 & 0.54 & 0.55 & 0.52 & 0.52 \\
$\log g_{2}(\mathrm{cgs})$ & 4.74 & 4.74 & 4.73 & 4.71 & 4.69 & 4.72 & 4.72 \\
$a\left(R_{\odot}\right)$ & 7.73 & 7.73 & 7.73 & 7.73 & 7.73 & 7.73 & 7.73 \\
$\Delta m$ & 0.875 & 0.893 & 0.872 & 0.832 & 0.794 & 0.891 & 0.888 \\
$i\left({ }^{\circ}\right)$ & 89.27 & 89.45 & 89.28 & 89.08 & 89.00 & 89.51 & 89.62 \\
$d(\mathrm{pc})$ & 42 & 42 & 42 & 42 & 42 & 42 & 41 \\
\hline
\end{tabular}

$V_{1}$ and $V_{2}$ are the theoretical velocities for the primary and the secondary at the orbital phase $\phi=0.7715$. All the solutions have the reduced $\chi^{2}$ the closest to 1 possible, for both data sets.

to be $\mathcal{L}_{3}=0.005 \pm 0.038$, and the corresponding best fit solution was found to be almost indistinguishable from the solution with $\mathcal{L}_{3}=0$.

To reproduce the observed radial velocities, we adjusted the semi-major orbital axis which, together with the orbital inclination and period, fixed the theoretical radial velocity amplitudes, $K_{1}$ and $K_{2}$ (in $\mathrm{km} \mathrm{s}^{-1}$ ). The apparent $V$-magnitude retrieved from SIMBAD is 11.10 mag. However, the Hipparcos measurement converted to Johnson's $V$ is equal to $11.73 \mathrm{mag}$, and our measurement is $11.78 \mathrm{mag}$ (Torres et al. 2006), the value adopted for the distance estimation. By using bolometric corrections for both components from Popper (1980), we can derive the absolute dimensions and, finally, estimate the distance to be $(42 \pm 3) \mathrm{pc}$. The interstellar absorption in such a short distance should be negligible. The parallax for the system measured by Hipparcos is $(22.79 \pm 4.87)$ mas, which infers $(44 \pm 9) \mathrm{pc}$, in good agreement with our determination.

All solutions of Table 4 are almost indistinguishable from the curve-fitting point of view, as can be seen by the rms of the residuals $(\sigma, 1 \mathrm{obs})$ in magnitudes. The probable intrinsic variability of one or both components makes it necessary to follow the system photometrically closely in time, preferably by observations in different passbands, and simultaneously with spectroscopic data to obtain radial velocity measurements. Only by obtaining more precise lightcurves in different photometric bands and a precise radial velocity curve will it be possible to eliminate this degeneracy in the solutions for the mass ratio. In this paper, we assume that the components are on the main-sequence, and make use of the mass-luminosity relation to fix $q$.

Figure 2 shows our solution for $q=0.9$ and Fig. 3 shows the corresponding residuals $\mathrm{O}-\mathrm{C}$. The steady and slow decreasing maxima found in ASAS observations was not taken into account in this analysis. It is common for $\mathrm{M}$ dwarfs to exhibit variations (e.g., Rockenfeller et al. 2006), mainly due to spots on their surfaces. However, we are unable to determine for the data that we analyzed, if the variability is caused by one or both eclipsing components. We searched for periodicities in the $\mathrm{O}-\mathrm{C}$ residuals between 0.08 day and 50 days, using the phase-dispersion method by Lafler \& Kinman (1965) and relative period steps of $\delta P / P=3 . \mathrm{d}-3$, and found a significant period of $(0.24965 \pm$ 0.00001 ) day (about $6 \mathrm{~h}$ ), as can be seen in Fig. 4. We note

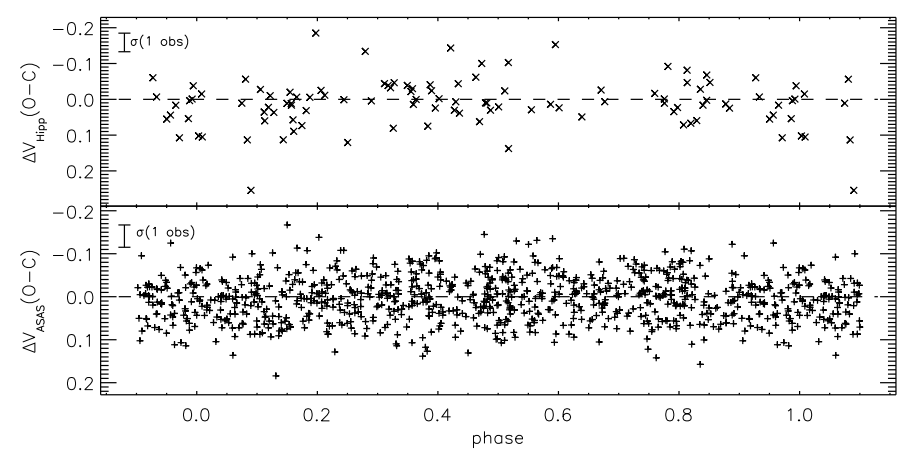

Fig. 3. The residuals O-C between the $V$ light curves of HIP $96515 \mathrm{~A}$ from Hipparcos ( $\times$ symbols) and ASAS (+ symbols) shown in Fig. 2, and our theoretical solution for $q=0.9$ obtained in Sect. 3.2. The standard error ( 1 observation) is shown in the upper left part of each panel.

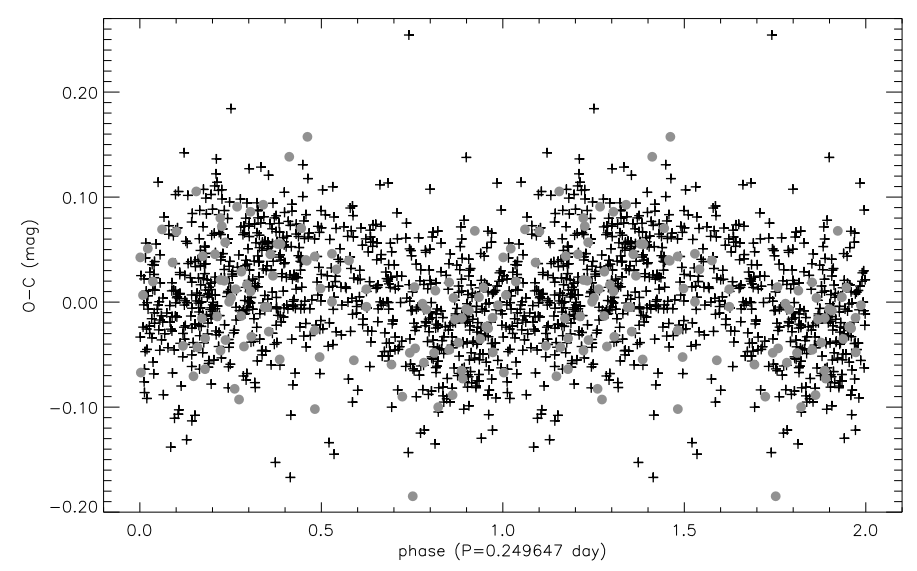

Fig. 4. The residuals O-C between the $V$ light curves of HIP $96515 \mathrm{~A}$ from ASAS (+ symbols) and Hipparcos (grey circles) shown in Fig. 3, folded with the period of $(0.24965 \pm 0.00001)$ day. Note that the pattern is present in both Hipparcos and ASAS data sets (two cycles are plotted).

that the variation pattern is present in both the Hipparcos and the ASAS data sets. The cadence of these observations (one or two observations a day, long intervals without observations being common) is inadequate for detecting these systematic variations in the $\mathrm{O}-\mathrm{C}$, which has the unfortunate result of introducing a multitude of aliases in the analysis. However, it is clear that the period of 0.24965 day, if real, is not the only one: Fig. 1 infers longer period variations, and we estimate in the $\mathrm{O}-\mathrm{C}$ at least another possible period of $(4100 \pm 200)$ days, but of low significance level due to the relatively short duration of the observations ( $\sim 6630$ days).

The period of 0.24965 day detected may be either due to pulsation or spots on the surface of one or both of the eclipsing components. Rockenfeller et al. (2006) listed other M dwarfs of $6 \mathrm{~h}$ period. However, if the variation is due to spots on HIP $96515 \mathrm{~A}$, it would indicate that the spotted component(s) spins significantly (more than a factor of 9) faster that the orbital motion (see e.g., CV Boo Torres et al. 2008b). This is probably not the case in HIP 96515 A: using an orbital period of 2.345 days, and the radius of the binary components from Table 5, we estimate rotational velocities of $\sim 14 \mathrm{~km} \mathrm{~s}^{-1}$ and $\sim 12 \mathrm{~km} \mathrm{~s}^{-1}$ for Aa and $\mathrm{Ab}$, respectively. These values are comparable with those derived from the optical spectrum (Table 3, and assuming an inclination of 89 degrees), indicating that the system is synchronized. 
Table 5. Preliminary physical parameters for HIP 96515 Aa\&Ab.

\begin{tabular}{lcc}
\hline \hline & Primary & Secondary \\
\hline \multicolumn{3}{l}{ Absolute dimension: } \\
Mass $\left(M_{\odot}\right)$ & $0.59 \pm 0.03$ & $0.54 \pm 0.03$ \\
Radius $\left(R_{\odot}\right)$ & $0.64 \pm 0.01$ & $0.55 \pm 0.03$ \\
$\log g($ c.g.s. $)$ & $4.59 \pm 0.03$ & $4.69 \pm 0.05$ \\
Photometric data: & \\
$\log T_{\text {eff }}(\mathrm{K})$ & $3.571 \pm 0.018$ & $3.555 \pm 0.019$ \\
$\log L / L_{\odot}$ & $-1.15 \pm 0.07$ & $-1.35 \pm 0.08$ \\
$M_{\text {bol }}$ & $7.63 \pm 0.18$ & $8.13 \pm 0.20$ \\
$M_{\mathrm{V}}$ & $9.10 \pm 0.18$ & $9.90 \pm 0.20$ \\
$L_{\text {sec }} / L_{\text {pri }}$ & \multicolumn{2}{c}{$0.48 \pm 0.24$} \\
$P_{\text {orb }}($ days $)$ & 2.3456 \\
$i($ deg $)$ & \multicolumn{2}{c}{$89.0 \pm 0.2$} \\
Distance $(\mathrm{pc})$ & \multicolumn{2}{c}{$42 \pm 3$} \\
\hline
\end{tabular}

Therefore, the origin of the 6h-period remains unclear with the current dataset.

By combining the results of Table 4, and taking into account the maximum variations in the variables along the grid, we can derive the preliminary absolute dimensions for HIP $96515 \mathrm{~A}$, which are listed in Table 5. The masses of the binary components, HIP $96515 \mathrm{Aa}$ and $\mathrm{Ab}$, are below $0.6 M_{\odot}$. Hence, HIP 96515 A is the fourteenth confirmed EB with component masses below $0.7 M_{\odot}$ (see e.g., Shkolnik et al. 2008). All these EBs are important when calibrating theoretical evolutionary models.

Finally, we note that the eclipsing system definitely deserves more observations, both multi-band photometry with a cadence that allows the determination of precise light curves, and spectroscopy, to obtain reliable radial velocity curves and spectral types for both components. If possible, HIP 96515 B, should also be observed simultaneously, to ensure consistency in the analysis.

\section{Characterization of HIP 96515 B}

We now focus on the characterization of HIP 96515 B. First, we investigated if the object forms a common proper-motion pair with HIP 96515 A by analyzing diffraction-limited infrared observations of the pair obtained in the course of our program to detect substellar objects around SACY targets (Huelamo et al. 2008 , in prep.). As a second step, we obtained an optical spectrum to derive the main properties of the object.

\subsection{NACO/VLT observations of HIP 96515}

We observed HIP 96515 twice with NAOS-CONICA (NACO), the Adaptive Optics facility at the Very Large Telescope (VLT), during the nights of the 2006 May 25 and 2008 June 16. The target was observed with the visible wavefront sensor and the $K_{\mathrm{s}}$-band filter. We used the S27 objective (nominal plate scale of $\sim 0.027^{\prime \prime} /$ pixel), which provides a total field-of-view of $\sim 27^{\prime \prime} \times$ $27^{\prime \prime}$. The total exposure time was $\sim 12$ min on source.

The data were obtained using a random jitter between the exposures to compute the sky emission. The images were reduced with the Eclipse reduction package (Devillard 1997) following the standard steps: dark-subtraction, flat-field division, and image shifting and stacking. The final image of HIP 96515 is displayed in Fig. 5. Apart from HIP 96515 A, we detected only the already known visual companion, HIP $96515 \mathrm{~B}$, at a projected separation of $8.6(\sim 378 \mathrm{AU}$ at $44 \mathrm{pc})$ from the primary. We
HIP 96515

NACO/Ks

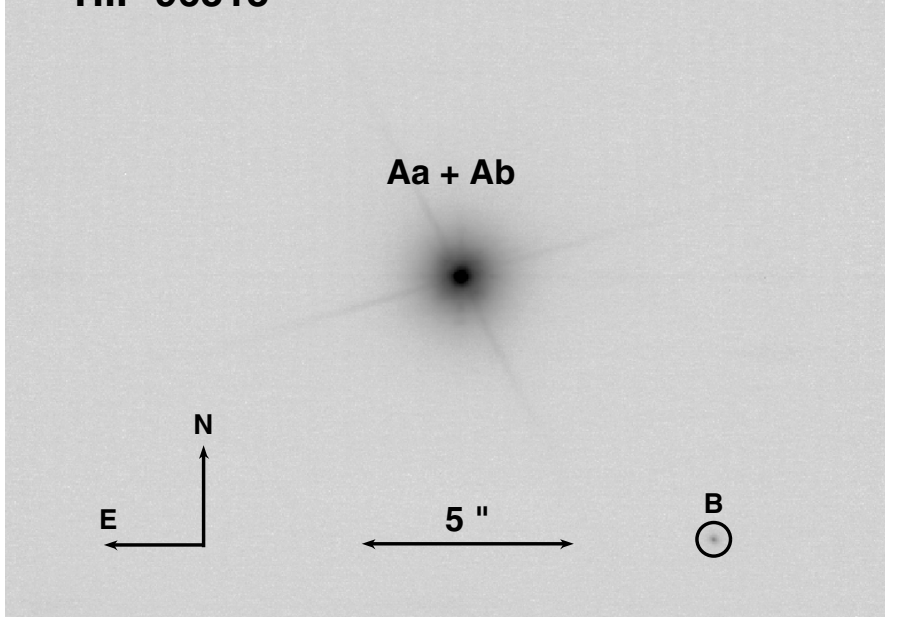

Fig. 5. VLT/NACO $K_{\mathrm{s}}$-band image of HIP $96515 \mathrm{~A}$ and its visual companion, HIP 96515 B, obtained in June 2008. The projected separation is of $378 \mathrm{AU}$ at a distance of $44 \mathrm{pc}$. Note that the eclipsing binary $(\mathrm{Aa} \& \mathrm{Ab})$ is not spatially resolved by NACO.

Table 6. Relative astrometry of HIP 96515 A \& B.

\begin{tabular}{llll}
\hline \hline Obs. Date & $\begin{array}{l}\text { Separation } \\
{[\operatorname{arcsec}]}\end{array}$ & $\begin{array}{l}\text { PA } \\
{[\mathrm{deg}]}\end{array}$ & Reference \\
\hline $2008-06-16$ & $8.586 \pm 0.008$ & $224.3 \pm 0.2$ & NACO/VLT, this paper \\
$2006-05-23$ & $8.586 \pm 0.010$ & $224.2 \pm 0.2$ & NACO/VLT, this paper \\
$2000-10-10$ & $8.3 \pm 0.2$ & 224.6 & 2MASS; Cutri et al. (2003) \\
1974.620 & 8.4 & 223 & Dommanget \& Nys (2000) \\
1910.705 & $8.9 \pm 0.3$ & 222 & Worley \& Douglass (1997) \\
\hline
\end{tabular}

note that the eclipsing binary is too tight to be resolved in the NACO images.

The difference in magnitude between HIP $96515 \mathrm{~A} \& \mathrm{~B}$ is $6.98 \pm 0.07 \mathrm{mag}$. Taking into account the brightness of the primary $\left(K_{\mathrm{s}}=7.9 \mathrm{mag}\right.$, see Table 2$)$, we estimate a $K_{\mathrm{s}}$-mag of $14.94 \pm 0.08$ for the secondary.

The NACO images were used to derive the separation and position angle of the two objects at two different epochs. To derive accurate astrometry of HIP $96515 \mathrm{~B}$ in the two images, we calibrated the plate scale and orientation of the infrared detector, CONICA, with the astrometric calibrator $\theta^{1}$ Ori $\mathrm{C}$ (McCaughrean \& Stauffer 1994). The results are displayed in Table 6 .

\subsection{HIP 96515 A \& B: a common proper-motion pair}

The astrometric accuracy of the NACO/VLT data is high enough to investigate if HIP 96515 A\&B are comoving. For completeness, we compared the NACO/VLT data with older (and less accurate) astrometric measurements from public surveys (e.g., Dommanget \& Nys 2000; Cutri et al. 2003). The visual binary is included in the Washington Double Star Catalog (WDSC, Worley \& Douglass 1997) with three observations between 1910 and 2000. Table 6 includes the angular separation and position angle of the two components at five different epochs. As can be seen, both remain almost constant during a time interval of 98 years.

Figure 6 (top panel) shows the difference in right ascension (RA) and declination (Dec) of the binary members measured at five epochs between 1910 and 2008. We have also 

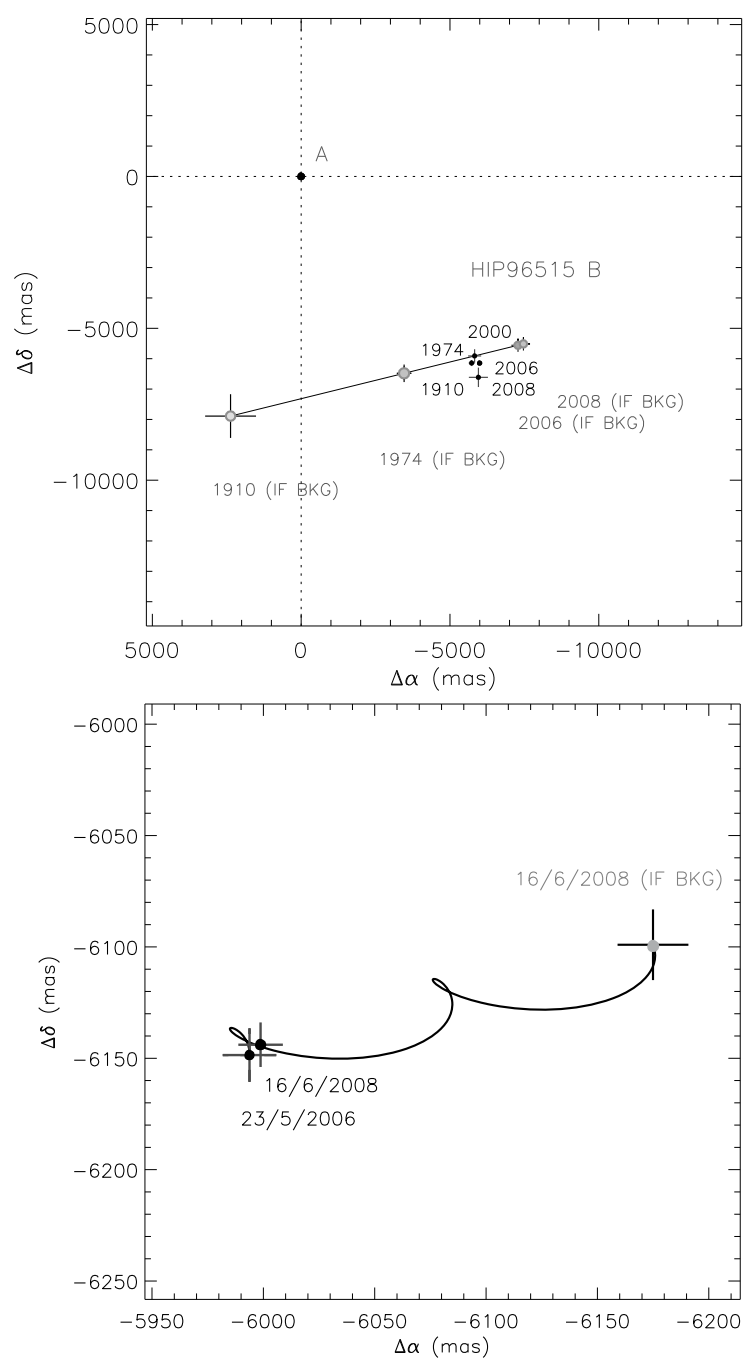

Fig. 6. Differences in right ascention and declination between HIP 96515 A \& B at different epochs (black circles). The expected position of a background object (grey circles and labels) at each epoch is also displayed. Top: all the astrometric data from 1910 to 2008. Bottom: only the NACO data, which provides the highest astrometric accuracy. The figure shows that the two objects are comoving, that is, they are a common proper motion pair.

overplotted the expected difference in RA and Dec of a background object taking into account the proper motion of the primary. If we focus on the NACO/VLT data only (Fig. 6, bottom panel), which has the highest astrometric accuracy, we can confirm that HIP96515 A \& B are comoving, that is, they form a common proper-motion pair.

\subsection{The optical spectrum of HIP 96515B: observations and modeling}

To ascertain the nature of HIP96515B, we obtained an optical spectrum of the target with the ESO Multi-Mode Instrument (EMMI) on the New Technology Telescope (NTT) on October 2007. The wavelength range of the spectrum was between $380 \mathrm{~nm}$ and $700 \mathrm{~nm}$, and the spectral resolution was $R \sim 1100$. The data were reduced with standard routines within IRAF, including bias subtraction and flat-fielding, and fluxcalibrated using a spectrophotometric standard observed during the night.
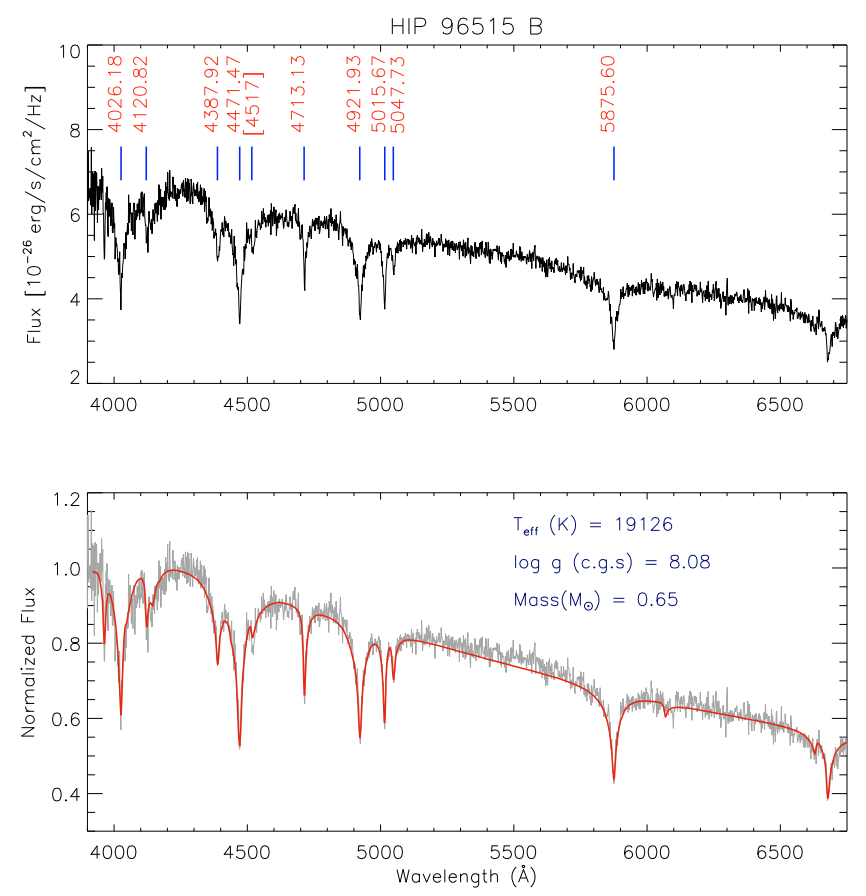

Fig. 7. Top: NTT/EMMI Optical spectrum of HIP 96515 B. We have marked all the identified He I lines. Bottom: fit of the optical spectrum using pure helium model atmospheres. The best fit parameters are displayed at the top right corner.

The optical spectrum of HIP 96515 B is displayed in Fig. 7. The continuum increases towards bluer wavelengths and the most obvious detected spectral features are the He I absorption lines, which are typical of helium (DB) white dwarfs.

We compared the optical spectrum of HIP 96515 B with atmospheric models of DB white dwarfs, to derive the physical parameters of the object. Our grid of model atmospheres and synthetic spectra for DB stars is described in Beauchamp et al. (1996). These include the improved Stark profiles of neutral helium from Beauchamp et al. (1997). The model atmospheres that we used here assume a pure helium composition and cover a range of effective temperatures of $T_{\text {eff }}=$ 10000 (1000), 16000 (2000), $30000 \mathrm{~K}$, and surface gravities of $\log g=7.0$ (0.5) 9.0. Our fitting technique relies on the nonlinear least squares method of Levenberg-Marquardt (Press et al. 1986), which is based on a steepest descent method. The model spectra (convolved with a Gaussian instrumental profile) and the optical spectrum were first normalized to a continuum defined to be unity. The calculation of $\chi^{2}$ was then completed for these normalized line profiles only. We adopt a pure helium composition based on the lack of any $\mathrm{H} \alpha$ absorption feature; additional models with mixed helium and hydrogen compositions allow us to set a limit of $N(\mathrm{H}) / N(\mathrm{He})<10^{-5}$.

As usual, there is a cool and a hot solution on each side of the temperature at which the line strengths reach their maximum, but the hot solution close to $T_{\text {eff }}=25000 \mathrm{~K}$ can easily be excluded based on the slope of the observed energy distribution. Our final solution, $T_{\text {eff }}=19130 \pm 190 \mathrm{~K}$ and $\log g=8.08 \pm 0.07$, is displayed in Fig. 7. These atmospheric parameters can be converted into a mass of $M=0.65 \pm 0.04 M_{\odot}$ using evolutionary models with $\mathrm{C} / \mathrm{O}$ cores, $q(\mathrm{He}) \equiv \log M_{\mathrm{He}} / M_{\star}=10^{-2}$, and $q(\mathrm{H})=10^{-10}$, which are representative of helium-atmosphere white dwarfs ${ }^{2}$.

\footnotetext{
${ }^{2}$ See

http://www . astro. umontreal. ca/ bergeron/CoolingModels
} 
Table 7. Best-fit parameters for HIP 96515 B.

\begin{tabular}{ll}
\hline \hline$T_{\text {eff }}(\mathrm{K})$ & $19126 \pm 195$ \\
$\log g(\mathrm{cgs})$ & $8.08 \pm 0.07$ \\
Mass $\left(M_{\odot}\right)$ & 0.648 \\
Radius $\left(R_{\odot}\right)$ & 0.01212 \\
$M_{V}(\mathrm{mag})$ & 10.89 \\
$M_{J}(\mathrm{mag})$ & 11.33 \\
distance $(\mathrm{pc})$ & 46 \\
Progenitor Mass $\left(M_{\odot}\right)$ & $3.1 \pm 0.8$ \\
WD Cooling age $(\mathrm{Myr})$ & $106 \pm 13$ \\
MS lifetime $(\mathrm{Myr})$ & $\sim 300^{1}$ \\
Total age $(\mathrm{Myr})$ & $\sim 400$ \\
\hline
\end{tabular}

${ }^{1}$ See text.

By using the best-fit parameters of the model, we can derive the absolute magnitudes in different photometric bands (see Table 7). Using $M_{\mathrm{J}}$ and the 2MASS $J$-band brightness of HIP $96515 \mathrm{~B}$, which is the measurement with the smallest photometric error, we estimate a distance to the source of $d=45.8 \mathrm{pc}$, which is in good agreement with both the Hipparcos parallax and the distance derived from the analysis of HIP $96515 \mathrm{~A}$.

The evolutionary sequences described above, as well as empirical relations (see e.g., Fontaine et al. 2001), allowed us to estimate the mass of the white dwarf progenitor and its cooling age. These data are provided in Table 7 . We obtained two different estimates of the white dwarf progenitor mass. First, we used the empirical initial-final mass relationship (IFMR) from Wood (1992), which is based on the study of the white dwarf luminosity function and mass distribution (see also Leggett et al. 1998; Fontaine et al. 2001). This yielded a value of $M_{\mathrm{MS}}=3.8 \pm$ $0.5 M_{\odot}$. Alternatively, we used the IFMR determined by Ferrario et al. (2005) based on white dwarfs observed in open clusters (see in particular their Fig. 1). This second relation yielded a significantly smaller final mass of $M_{\mathrm{MS}}=2.5 \pm 0.5 M_{\odot}$. However, as discussed by Ferrario et al., the IMFR of most clusters appears to have an intrinsic spread (i.e., there is a range of initial masses that gives rise to a given final mass). Hence, a value of $M_{\mathrm{MS}}$ as high as $3.8 M_{\odot}$ could not be necessarily ruled out. In the following, we thus adopt a mean value of $M_{\mathrm{MS}}=3.1 \pm 0.8 M_{\odot}$ to allow for the possible mass range of the progenitor.

We derived the main-sequence lifetime of HIP 96515 B using two sets of evolutionary tracks for a $3.1 \pm 0.8 M_{\odot}$ star. First, we used the ATON 2.4 models, which are a modified version of models by D'Antona \& Mazzitelli (D'Antona \& Mazzitelli 1994; D'Antona \& Mazzitelli 1997), and are fully described in Landin et al. (2006). According to these evolutionary models, a $3.1 \pm 0.8 M_{\odot}$ star spends $280_{-125}^{+346} \mathrm{Myr}$ on the main-sequence, i.e., from the zero-age main-sequence (ZAMS) to the terminal age main-sequence (TAMS). On the other hand, Claret (2004) models $(Z=0.02)$ predict a span of $\sim 317 \mathrm{Myr}$ for the MS time of a 3.1 $M_{\odot}$. Assuming a MS lifetime of $\sim 300 \mathrm{Myr}$ and a cooling time of $\sim 100 \mathrm{Myr}$ (see Table 7), the total age of the system would be $\sim 400 \mathrm{Myr}$, where the errors are dominated by the uncertainty in the mass.

\section{Discussion}

The comparison of HIP $96515 \mathrm{Aa} \& \mathrm{Ab}$ with evolutionary tracks by Baraffe et al. (1998) provides an age of 50 Myr for masses of $0.5 M_{\odot}$ (with alpha $\left.=1\right)$, and places the binary on the PMS (see Fig. 8). The PMS nature of HIP 96515 A is also supported by comparison with tracks generated by the ATON 2.4 stellar

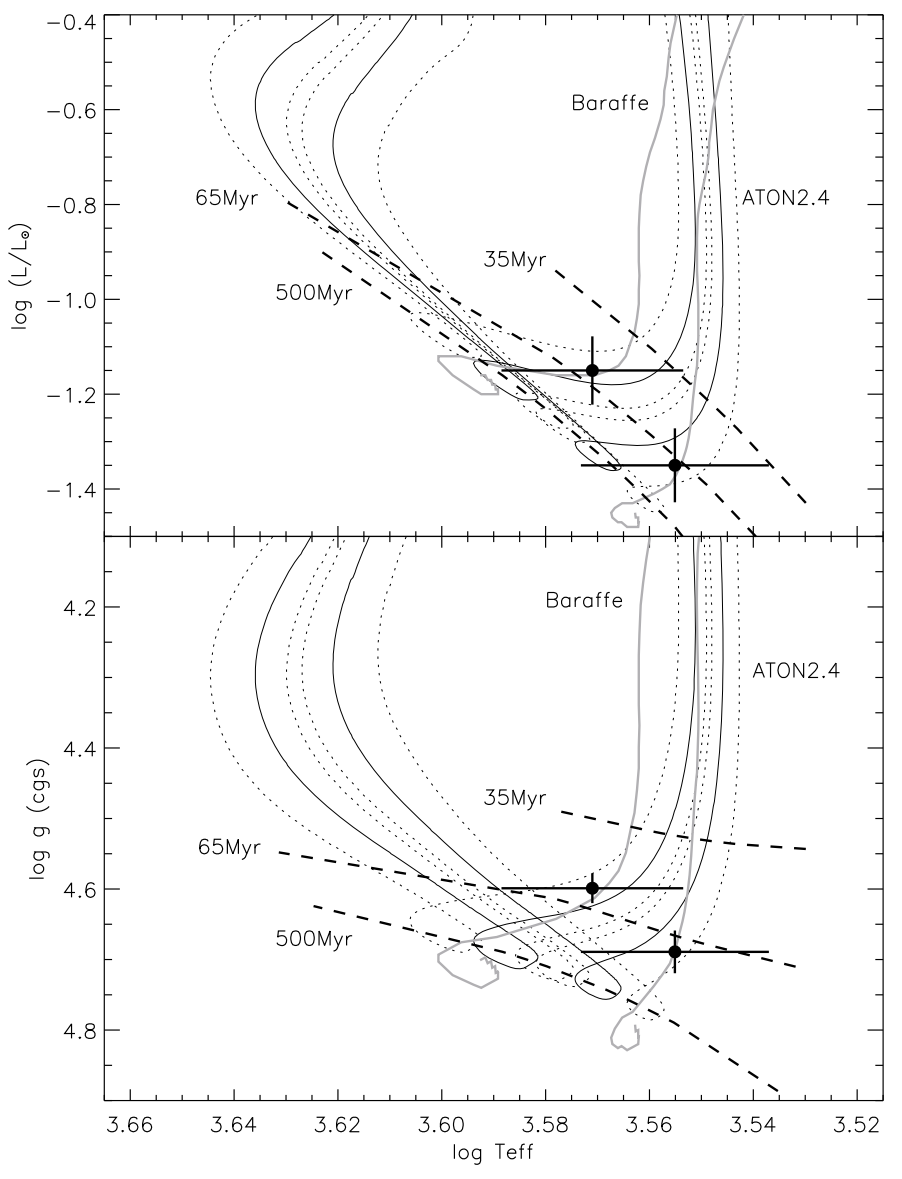

Fig. 8. Stellar luminosity (top panel) and surface gravity (bottom panel) versus effective temperature. HIP $96515 \mathrm{Aa}$ and $\mathrm{Ab}$ are represented by filled circles. The grey and black solid lines represent evolutionary tracks by Baraffe et al. (1998) and Landin et al. (2006), respectively, for 0.6 and $0.5 M_{\odot}$ stellar masses. The dotted lines represent the uncertainties in the stellar masses for the ATON models. The comparison of HIP 96515 A with the two sets of evolutionary tracks provides an age of $\sim 60 \mathrm{Myr}$, that is, places the eclipsing binary members on the pre-main sequence, although with large uncertainties.

evolutionary code, where we adopted the solar chemical composition $(Z=0.0175$ and $Y=0.27)$ and alpha equal to 1 . The tracks in Fig. 8 generated with the ATON2.4 code cover the PMS, MS and earlier stages of post main-sequence phases. The most probable age, according to ATON 2.4, is 60 Myr (Fig. 8), although with large uncertainties.

On the other hand, our astrometric study shows that the white dwarf and the EB are co-moving. If we assume that HIP 96515 A\&B are coeval, the age of the triple system should be $\sim 400 \mathrm{Myr}$, which is significantly older than the age provided by the PMS tracks for HIP $96515 \mathrm{~A}$, although within the observational uncertainties of the primary star (see Fig. 8).

To shed light on the evolutionary status of HIP 96515 A, we have studied the kinematical properties of the object. Using an average distance of $45 \mathrm{pc}$ (from the EB and WD independent studies presented here), the RV derived by Torres et al. (2006), and the proper motions measured by Hipparcos (see Table 1), we have derived the $U V W$ components of the Galactic space velocity vector: $U V W=-6.0,-0.5,-21.6 \mathrm{~km} \mathrm{~s}^{-1}$. We have compared these values with the $U V W$ vectors of young moving groups in the solar neighborhood included in Zuckerman \& Song (2004) and Torres et al. (2008a). We conclude that HIP96515 A does not share the kinematical properties of any of these associations. 


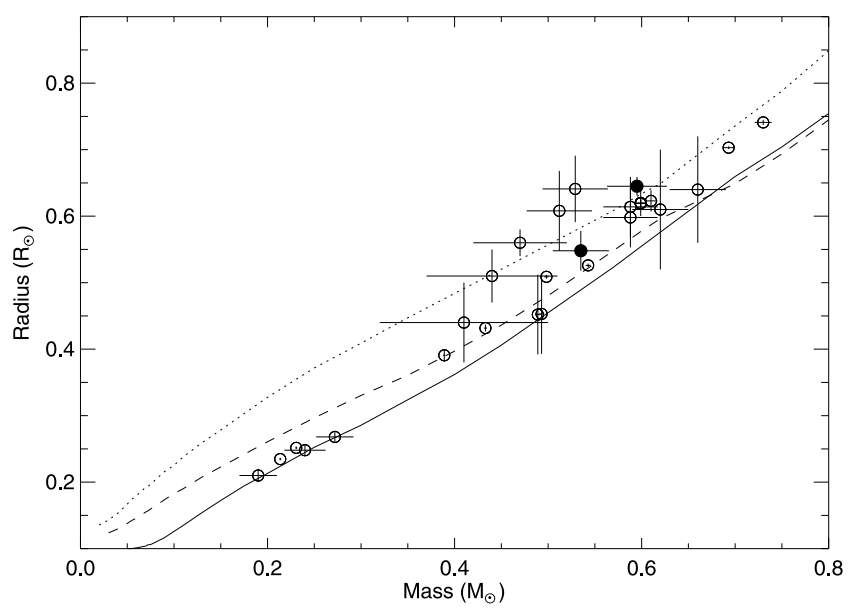

Fig. 9. Mass-luminosity relation for all known eclipsing binaries with masses $M<0.7 M_{\odot}$ (see Shkolnik et al. 2008, and references therein). HIP $96515 \mathrm{Aa}$ and $\mathrm{Ab}$ are represented by filled circles. The dotted, dashed, and solid lines represent evolutionary tracks by Baraffe et al. (1998) for 50, 100, and $500 \mathrm{Myr}$, respectively.

Assuming an age of $400 \mathrm{Myr}$ for the triple system, a possible explanation of the age of 60 Myr derived from PMS evolutionary tracks is the different evolution of both EBs and very magnetically active stars, and non-active objects. EBs are normally fast rotators ( $P_{\text {rot }}<3$ days) and exhibit strong X-ray emission related to the presence of magnetic fields (López-Morales 2007). As explained by e.g., Chabrier et al. (2007), rapid rotation and/or magnetic fields reduce the efficiency of large-scale thermal convection in their interior leading to less efficient heat transport. The reduction in the area of the stellar radiating surface due to the presence of cold spots can cause lower values of $T_{\text {eff }}$ and larger values of the radius, thus departing significantly from model predictions (see e.g., Torres \& Ribas 2002).

To investigate this problem in more detail, we have studied the radius vs. mass relation for HIP $96515 \mathrm{~A}$ and compared this with the predictions of evolutionary models by Baraffe et al. (1998). The adopted models correspond to a solar metallicity of $[\mathrm{Fe} / \mathrm{H}]=0$, and a mixing length of $\alpha=1$. The results are displayed in Fig. 9, which includes all the low-mass eclipsing binaries confirmed so far. The data was adopted from Shkolnik et al. (2008) and references therein.

By assuming an age of $400 \mathrm{Myr}$ for the triple system, the radii of HIP $96515 \mathrm{Aa} \& \mathrm{Ab}$ were found to be $\sim 15 \%$ and $10 \%$ larger, respectively, than predicted by the evolutionary tracks (see Fig. 9). These discrepancies were previously observed in similar eclipsing binaries and attributed to the effect of rapid rotation, magnetic activity, and/or metallicity (e.g., Berger et al. 2006; Chabrier et al. 2007; López-Morales 2007).

\section{Results and conclusions}

We have studied in detail HIP 96515, a triple system consisting of a double-lined spectroscopic binary (SB2) and a comoving white dwarf with a projected separation of $8^{\prime \prime} .6$. We have analyzed both new and archival observations of the system. Our main results can be summarized as follows:

- The analysis of multi-epoch optical photometry of HIP 96515 A has revealed that this SB2 is an eclipsing binary with almost equal-mass components: $M_{A a}=0.59 M_{\odot}$ and $M_{A b}=0.54 M_{\odot}$. This is the fourteenth confirmed eclipsing binary with component masses below $0.7 M_{\odot}$, all of them being of extreme importance to calibrate theoretical evolutionary tracks.

- Archival astrometry and new NACO/VLT data show that HIP 96515 A\&B is a common proper-motion pair.

- The optical spectrum of HIP96515 B is consistent with that of a hot DB white dwarf. We have modeled the spectrum using evolutionary cooling sequence models, deriving a temperature, surface gravity, and mass of $T_{\text {eff }}(\mathrm{K})=19126$, $\log g=8.08$, and $M=0.65 M_{\odot}$, respectively. Empirical relations provide a progenitor mass of $3.1 \pm 0.8 M_{\odot}$, and a white dwarf cooling age of $100 \pm 13$ Myr. The MS lifetime of a $3.1 M_{\odot}$ star, as derived from evolutionary models, is $\sim 300 \mathrm{Myr}$, which corresponds to a total age of $\sim 400 \mathrm{Myr}$ for the white dwarf.

- A direct comparison of the EB members with evolutionary tracks (Baraffe et al. 1998; Landin et al. 2006) places them on the PMS with an age of $~ 60 \mathrm{Myr}$, although with large uncertainties. We note that HIP $96515 \mathrm{~A}$ does not share the kinematical properties of known young moving groups in the solar vicinity.

- If HIP 96515 A\&B are coeval, the system must be older than $300 \mathrm{Myr}$. The discrepancy between this age and the $60 \mathrm{Myr}$ provided by PMS tracks might be explained by the different evolution of EBs, which normally exhibit fast rotation and stronger magnetic fields than non-magnetic stars. Since HIP 96515 A shows fast rotation and strong X-ray emission, it can depart from the predictions of theoretical models, which place the binary on the PMS.

- If we assume an age of $\sim 400 \mathrm{Myr}$ for the triple system, the comparison of HIP 96515 Aa\&Ab with evolutionary tracks by Baraffe et al. (1998) shows that the models underestimate the stellar radii by $15 \%$ and $10 \%$, for the primary and the secondary, respectively. This behavior has been previously observed in similar low-mass EBs.

Finally, we note that additional RV observations are needed to constrain more robustly the orbital and physical parameters of the eclipsing binary.

Acknowledgements. We are very grateful to I. Saviane for obtaining the NTT/EMMI spectrum presented here. N.H. is indebted to the Spanish Programa Juan de la Cierva. This research has been funded by Spanish grant MEC ESP2007-65475-C02-02 and MEC/Consolider-CSD2006-0070. N.H. and C.T. gratefully acknowledge support from ESO-DGDF 2007 program. G.C. acknowledges support from the Faculty of the European Space Astronomy Centre (ESAC). H.B. acknowledges the funding from the European Commission's Sixth Framework Program as a Marie Curie Outgoing International Fellow (MOIFCT-2005-8389). L.P.R.V. and N.R.L. gratefully acknowledge financial support from the Brazilian agencies CAPES, CNPq and FAPEMIG. P.B. is a Cottrell Scholar of the Research Corporation for Science Advancement and is supported in part by the NSERC Canada and by the Fund FQRNT(Québec). This research has made use of the SIMBAD database, operated at CDS, Strasbourg, France, of NASA's Astrophysics Data System Abstract Service, and of the Washington Double Star Catalog maintained at the US Naval Observatory.

\section{References}

Alencar, S. H. P., \& Vaz, L. P. R. 1997, A\&A, 326, 257

Alencar, S. H. P., Vaz, L. P. R., \& Nordlund, A. 1999, A\&A, 346, 556

Allard, F., \& Hauschildt, P. H. 1995, ApJ, 445, 433

Allard, F., Hauschildt, P. H., Alexander, D. R., \& Starrfield, S. 1997, ARA\&A, 35,137

Baraffe, I., Chabrier, G., Allard, F., \& Hauschildt, P. H. 1998, A\&A, 337, 403

Baraffe, I., Chabrier, G., Barman, T. S., Allard, F., \& Hauschildt, P. H. 2003, A\&A, 402, 701

Barrado y Navascués, D., Stauffer, J. R., Song, I., \& Caillault, J.-P. 1999, ApJ, 520, L123 
Basri, G., Marcy, G. W., \& Graham, J. R. 1996, ApJ, 458, 600

Beauchamp, A., Wesemael, F., Bergeron, P., Liebert, J., \& Saffer, R. A. 1996, in Hydrogen Deficient Stars, ed. C. S. Jeffery, \& U. Heber, ASP Conf. Ser., 96, 295

Beauchamp, A., Wesemael, F., \& Bergeron, P. 1997, ApJS, 108, 559

Berger, D. H., Gies, D. R., McAlister, H. A., et al. 2006, ApJ, 644, 475

Chabrier, G., Baraffe, I., Allard, F., \& Hauschildt, P. 2000, ApJ, 542, 464

Chabrier, G., Gallardo, J., \& Baraffe, I. 2007, A\&A, 472, L17

Chauvin, G., Thomson, M., Dumas, C., et al. 2003, A\&A, 404, 157

Claret, A. 2000, A\&A, 363, 1081

Claret, A. 2004, A\&A, 424, 919

Cutri, R. M., Skrutskie, M. F., van Dyk, S., et al. 2003, VizieR Online Data Catalog, 2246, 0

D'Antona, F., \& Mazzitelli, I. 1994, ApJS, 90, 467

D’Antona, F., \& Mazzitelli, I. 1997, Mem. Soc. Astron. Ital., 68, 807

Devillard, N. 1997, The Messenger, 87, 19

Dommanget, J., \& Nys, O. 2000, A\&A, 363, 991

Ferrario, L., Wickramasinghe, D., Liebert, J., \& Williams, K. A. 2005, MNRAS, 361,1131

Fontaine, G., Brassard, P., \& Bergeron, P. 2001, PASP, 113, 409

Hauschildt, P. H., Allard, F., Alexander, D. R., \& Baron, E. 1997a, ApJ, 488, 428

Hauschildt, P. H., Baron, E., \& Allard, F. 1997b, ApJ, 483, 390

Kenyon, S. J., \& Hartmann, L. 1995, ApJS, 101, 117

Lafler, J., \& Kinman, T. D. 1965, ApJS, 11, 216

Lafreniere, D., Doyon, R., Marois, C., et al. 2007, arXiv e-prints, 705

Landin, N. R., Ventura, P., D’Antona, F., Mendes, L. T. S., \& Vaz, L. P. R. 2006 A\&A, 456, 269

Leggett, S. K., Ruiz, M. T., \& Bergeron, P. 1998, ApJ, 497, 294

López-Morales, M. 2007, ApJ, 660, 732

Lowrance, P. J., Becklin, E. E., Schneider, G., et al. 2005, AJ, 130, 1845

Masciadri, E., Mundt, R., Henning, T., Alvarez, C., \& Barrado y Navascués, D. 2005, ApJ, 625, 1004

McCaughrean, M. J., \& Stauffer, J. R. 1994, AJ, 108, 1382
Neuhäuser, R. 1997, Science, 276, 1363

Paczyński, B., Szczygieł, D. M., Pilecki, B., \& Pojmański, G. 2006, MNRAS, 368, 1311

Perryman, M. A. C., Lindegren, L., Kovalevsky, J., et al. 1997, A\&A, 323, L49

Pojmanski, G., \& Maciejewski, G. 2005, Acta Astron., 55, 97

Popper, D. M. 1980, ARA\&A, 18, 115

Press, W. H., Flannery, B. P., \& Teukolsky, S. A. 1986, Numerical recipes, The art of scientific computing (Cambridge: University Press)

Rockenfeller, B., Bailer-Jones, C. A. L., \& Mundt, R. 2006, A\&A, 448, 1111

Schmitt, J. H. M. M., Fleming, T. A., \& Giampapa, M. S. 1995, ApJ, 450, 392

Shkolnik, E., Liu, M. C., Reid, I. N., et al. 2008, ApJ, 682, 1248

Stelzer, B., \& Neuhäuser, R. 2001, A\&A, 377, 538

Torres, G., \& Ribas, I. 2002, ApJ, 567, 1140

Torres, C. A. O., Quast, G. R., de La Reza, R., et al. 2003, in Open issues in Local Star Formation, ed. J. Lépine, \& J. Gregorio-Hetem, Astrophys. Space Sci. Lib., 299, 83

Torres, C. A. O., Quast, G. R., da Silva, L., et al. 2006, A\&A, 460, 695

Torres, C. A. O., Quast, G. R., Melo, C. H. F., \& Sterzik, M. F. 2008a, Handbook of Star Forming Regions, Vol. II, The Southern Sky, ASP, Monograph 5, ed. Bo Reipurth

Torres, G., Vaz, L. P. R., \& Sandberg Lacy, C. H. 2008b, arXiv e-prints

Urban, S. E., Corbin, T. E., Wycoff, G. L., et al. 1998, AJ, 115, 1212

van Leeuwen, F. 2007, A\&A, 474, 653

Vaz, L. P. R., Andersen, J., \& Claret, A. 2007, A\&A, 469, 285

Voges, W., Aschenbach, B., Boller, T., et al. 1999, A\&A, 349, 389

Wilson, R. E. 1979, ApJ, 234, 1054

Wilson, R. E. 1993, in New Frontiers in Binary Star Research, ed. K.-C. Leung,

\& I.-S. Nha, ASP Conf. Ser., 38, 91

Wilson, R. E., \& Devinney, E. J. 1971, ApJ, 166, 605

Wood, M. A. 1992, ApJ, 386, 539

Worley, C. E., \& Douglass, G. G. 1997, A\&AS, 125, 523

Zahn, J.-P. 1989, A\&A, 220, 112

Zuckerman, B., \& Song, I. 2004, ARA\&A, 42, 685 